\title{
Invariant curves around a parabolic fixed point at infinity
}

\author{
DOV AHARONOV AND URI ELIAS \\ Department of Mathematics, Technion, Israel Institute of Technology, \\ Haifa 32000, Israel
}

(Received 12 April 1988, revised 26 July 1988)

Abstract. The stability of a fixed point of an area-preserving transformation in the plane is characterized by the invariant curves which surround it. The existence of invariant curves had been extensively studied for elliptic fixed points. Here we study the similar problem for parabolic fixed points. In particular we are interested in the case where the fixed point is at infinity.

\section{Introduction}

Consider an area-preserving mapping of the plane which has a fixed point and let $\lambda_{1}, \lambda_{2}$ be the eigenvalues of its Jacobian at this point. Since the mapping is area preserving, the determinant of the Jacobian is 1 and it follows that $\lambda_{1} \lambda_{2}=1$. Since the mapping is real, $\lambda_{1}, \lambda_{2}$ are either both real or are complex conjugates. If $\lambda_{1}, \lambda_{2}$ are real and not \pm 1 , the fixed point is called hyperbolic and it cannot be stable. If $\lambda_{i}=\bar{\lambda}_{2},\left|\lambda_{1}\right|=\left|\lambda_{2}\right|=1$ and $\lambda_{1}, \lambda_{2}$ are not \pm 1 , the fixed point is called elliptic. This case is extensively studied and it is known that under some mild conditions the fixed point is stable and it is surrounded by closed invariant curves $[6,7,10]$. Numerical examples are found in [2-4]. Finally, if $\lambda_{1}=\lambda_{2}=1$, the fixed point is called parabolic. This case attracted less attention, probably because it is a degenerate one. This will be the subject of the present work.

We consider an area-preserving mapping

$$
\begin{aligned}
& x_{1}=x+P(x, y), \\
& y_{1}=y+Q(x, y),
\end{aligned}
$$

where $P, Q=o(r)$ as $r=\left(x^{2}+y^{2}\right)^{1 / 2} \rightarrow 0$. Here $(x, y)=(0,0)$ is a parabolic fixed point with eigenvalues $\lambda_{1}=\lambda_{2}=1$. Such a degenerate fixed point may be stable or nonstable. For example, the mapping

$$
\begin{aligned}
& x_{1}=x+f(y), \\
& y_{1}=y+g\left(x_{1}\right),
\end{aligned}
$$

is area preserving; however, if $f, g$ vanish at 0 and are positive elsewhere, the iterated mappings of each $\left(x_{0}, y_{0}\right), x_{0}, y_{0}>0$, diverge to infinity. The present work discusses when parabolic fixed points are surrounded by closed invariant curves and exhibit stable behaviour. 
The main tool to establish the existence of invariant curves is Moser's theorem about twist mappings [6, Theorem $3 ; 10, \S 32]$ :

THEOREM. Given the mapping

$$
\begin{aligned}
& \theta_{1}=\theta+\gamma \rho+f(\rho, \theta), \\
& \rho_{1}=\rho+g(\rho, \theta),
\end{aligned}
$$

in the annulus $a<\rho<b,-\infty<\theta<\infty, b-a \geq 1$, where $f, g$ are real-analytic and periodic in $\theta$ and every closed curve sufficiently close to $\rho=$ constant intersects its image curve. For $\varepsilon>0$ there exists $\delta$, independent of $\gamma$, such that if

$$
|f|+|g|<\gamma \delta,
$$

then the mapping admits infinitely many invariant curves of the form

$$
\rho=v(t), \quad \theta=t+u(t),
$$

with $y, v$ real-analytic, periodic functions and $|u(t)|,|v(t)-\omega|<\varepsilon$.

The assumption that $f, g$ are real analytic is not essential. In [9] it is only assumed that $f, g \in C^{5}$, and in [5] that $f, g \in C^{3+\alpha}$.

We shall show that under suitable assumptions the mapping (1.1) can be transformed into a twist mapping to which the theory of Moser may be applied.

Most of this work deals with parabolic fixed points at infinity. However, we begin it in $\$ 2$ with a result about a finite fixed point, which motivates the rest of the discussion. $\S 3$ deals with a parabolic fixed point at infinity and explains how it differs from a finite fixed point. We discuss the mapping when its behaviour is determined by the first nonlinear terms and distinguish between the cases when these terms are homogeneous of order $h, h \leq-2$, and when they are of order $h=-1$. In $\S 4$ we study a mapping which is analytic but its dominant part is only piecewise continuous at infinity.

The most interesting mappings are, of course, the area-preserving ones. However, because of reasons which will be clarified later on, we prefer to mention first a result which emphasizes the intersection property rather than the area-preserving property. For an area-preserving mapping this result is a particular case of a more general theorem of Simo [11] and we also use the same ideas. $\dagger$

\section{Invariant curves around a finite fixed point}

THEOREM 1. Given a real-analytic mapping

$$
\begin{aligned}
& x_{1}=x+P(x, y), \\
& y_{1}=y+Q(x, y),
\end{aligned}
$$

in a neighborhood of $(0,0)$, which can be written as

$$
\begin{aligned}
& x_{1}=x+p(x, y)+\hat{p}(x, y) \\
& y_{1}=y+q(x, y)+\hat{q}(x, y),
\end{aligned}
$$

† The authors wish to express their gratitude to the referee who drew their attention to Simo's work. 
where $p(x, y), q(x, y)$ are polynomials homogeneous of degree $h, h>1$, and $\hat{p}, \hat{q}$ are such that

$$
\hat{p}(x, y), \hat{q}(x, y)=O\left(r^{h+1}\right) \quad \text { near } r=0 .
$$

The fixed point $(0,0)$ is surrounded by closed invariant curves provided the following assumptions hold:

$$
\begin{gathered}
x q(x, y)-y p(x, y) \neq 0 \text { for }(x, y) \neq(0,0), \\
p_{x}+q_{y}=0,
\end{gathered}
$$

(c) Every closed curve sufficiently close to $x q-y p=$ constant intersects its image curve.

If (2.1) is area preserving, then assumptions (b) and (c) hold true automatically. Proof. First we present a full proof of this simple result since it motivates the rest of the work and then describe its relation to [11].

The differential system

$$
\begin{aligned}
& x^{\prime}=p(x, y), \\
& y^{\prime}=q(x, y),
\end{aligned}
$$

is a Hamiltonian system with

$$
H(x, y)=[x q(x, y)-y p(x, y)] /(h+1) .
$$

Indeed, by the homogeneity of degree $h$ of $q, x q_{x}+y q_{y}=h q$, and by (2.5),

$$
(h+1) H_{x}=\left(x q_{x}+q\right)-y p_{x}=\left(x q_{x}+y q_{y}\right)+q=(h+1) q, \quad H_{y}=-p .
$$

We may assume, for example, that

$$
H(x, y)>0 \text { for }(x, y) \neq(0,0) .
$$

Let us define

$$
\begin{aligned}
& \rho=H(x, y)^{(n-1) /(h+1)}, \\
& \theta=\int_{0}^{\operatorname{arctg}(y / x)} H(\cos \phi, \sin \phi)^{-2 /(h+1)} d \phi,
\end{aligned}
$$

where the values of arctg pass from one branch to another as $(x, y)$ surrounds the origin.

Since $H(x, y) \neq 0$ for $(x, y) \neq(0,0)$ and $H(x, y)$ is a homogeneous polynomial, $H(x, y)=c$ are closed curves for $c>0$. They are also starlike since

$$
(d / d t) \operatorname{arctg}(y / x)=\left(y^{\prime} x-x^{\prime} y\right) /\left(x^{2}+y^{2}\right)=(x q-y p) / r^{2} \neq 0 .
$$

Thus $H(x, y)=c$ is a family of closed, starlike, similar curves which cover $R^{2} \backslash\{0\}$. Now, $\rho$ increases along every ray through $(0,0)$ and $\theta$ increases with $\arg (y / x)$, hence the correspondence $(x, y) \leftrightarrow(\rho, \theta)$ is locally $1: 1$ in $R^{2} \backslash\{0\}$. When one returns to a point after surrounding the origin, $\theta$ is increased by

$$
\omega=\int_{0}^{2 \pi} H(\cos \phi, \sin \phi)^{-2 /(h+1)} d \phi,
$$

so our change of variables is even globally one-to-one. $\rho, \theta$ are, of course, real analytic functions of $x, y$ for $(x, y) \neq(0,0)$. 
Our aim is to prove that under this change of variables, the mapping (2.1) becomes the twist mapping

$$
\begin{aligned}
& \theta_{1}=\theta+\rho+f(\rho, \theta), \\
& \rho_{1}=\rho+g(\rho, \theta),
\end{aligned}
$$

where $f(\rho, \theta), g(\rho, \theta)$ are real-analytic in the domain $\rho>0$, periodic in $\theta$ and

$$
f=o(\rho), g=o\left(\rho^{2}\right) \quad \text { as } \rho \rightarrow 0^{+} .
$$

Let us denote $\Delta x=x_{1}-x=P, \Delta y=y_{1}-y=Q$ and analogously $\Delta \rho=\rho_{1}-\rho, \Delta \theta=$ $\theta_{1}-\theta$. Then, near the origin we have by our previous calculations

$$
\begin{aligned}
\rho_{1}-\rho=\Delta \rho & =\frac{h-1}{h+1} H^{[(h-1) /(h+1)-1]}\left[H_{x} \Delta x+H_{y} \Delta y+\cdots\right] \\
& =\frac{h-1}{h+1} H^{-2 /(h+1)}[q P-p Q+\cdots] \\
& =\frac{h-1}{h+1} H^{-2 /(h+1)}[q \hat{p}-p \hat{q}+\cdots]=H^{-2 /(h+1)} O\left(r^{2 h+1}\right),
\end{aligned}
$$

since the other terms are $\frac{1}{2}\left[H_{x x} p^{2}+\cdots\right]=O\left(r^{3 h-1}\right)$. But now $H(x, y)$ is a homogeneous polynomial of order $h+1$ and $H(x, y) \neq 0$ for $(x, y) \neq(0,0)$, therefore $H(x, y)>A r^{h+1}$. So (2.11) becomes

$$
H^{-2 /(h+1)} O\left(H^{(2 h+1) /(h+1)}\right)=O\left(H^{(2 h-1) /(h+1)}\right)=o\left(H^{2(h-1) /(h+1)}\right)=o\left(\rho^{2}\right) .
$$

Hence $\rho_{1}=\rho+g(\rho, \theta)$ where $g(\rho, \theta)=o\left(\rho^{2}\right)$ near $\rho=0$. As for $\theta$,

$$
\begin{aligned}
\theta_{1}-\theta=\Delta \theta & =\Delta(\operatorname{arctg}(y / x)) H(\cos \phi, \sin \phi)^{-2 /(h+1)} \\
& =\frac{x \Delta y-y \Delta x}{r^{2}} H(x / r, y / r)^{-2 /(h+1)}(1+o(1)) \\
& =[x(q+\hat{q})-y(p+\hat{p})] H(x, y)^{-2 /(h+1)}(1+o(1)) .
\end{aligned}
$$

by our assumptions $x \hat{q}-y \hat{p}=O\left(r^{h+2}\right)$ while $x q-y p \geq A r^{h+1}$. Hence $x \hat{q}-y \hat{p}=$ $o(x q-y p)$ and

$$
\theta_{1}-\theta=(x q-y p) H^{-2 /(h+1)}(1+o(1))=H^{1-2 /(h+1)}(1+o(1))=\rho(1+o(1)) .
$$

Thus $\theta_{1}=\theta+\rho+f(\rho, \theta)$, where $f(\rho, \theta)=o(\rho)$ near $\rho=0 . f(\rho, \theta)$ and $g(\rho, \theta)$ are obviously analytic when $\rho>0$ and have period $\omega$, so (2.9)-(2.10) are established.

A small neighbourhood $0<H(x, y)<\varepsilon^{(h+1) /(h-1)}$ of the origin is thus mapped on a narrow annulus $0<\rho<\varepsilon$. When we replace $\rho$ by $\varepsilon \rho$, we get

$$
\begin{aligned}
& \theta_{1}=\theta+\varepsilon \rho+\tilde{f}, \\
& \rho_{1}=\rho+\tilde{g} .
\end{aligned}
$$

Here $0<\rho<1$ and $\tilde{f}(\rho, \theta)=f(\varepsilon \rho, \theta)=o(\varepsilon), \tilde{g}(\rho, \theta)=\varepsilon^{-1} g(\varepsilon \rho, \theta)=o(\varepsilon)$. Hence $(|\tilde{f}|+|\tilde{g}|) / \varepsilon \rightarrow 0$ as $\varepsilon \rightarrow 0$ as required in the theory of Moser. Finally, in order to use Moser's theorem, we have only to guarantee that curves which are sufficiently close to the circles $\rho=$ constant, intersect their image curves. This is ensured by (c) and the proof is completed. 
Note that if (2.1) is area preserving then (b) and (c) are always satisfied. For the Jacobian of (2.2) is

$$
J=\left|\begin{array}{cc}
1+p_{x}+\hat{p}_{x} & p_{y}+\hat{p}_{y} \\
q_{x}+\hat{q}_{x} & 1+q_{y}+\hat{q}_{y}
\end{array}\right|=1+p_{x}+q_{y}+o\left(r^{h}\right) .
$$

Since $J \equiv 1$ and $p_{x}+q_{y}$ is homogeneous of degree $h-1$, we must have

$$
p_{x}+q_{y}=0 .
$$

Also, if an area-preserving mapping has a finite fixed point, then every closed curve with surrounds this fixed point intersects its image.

When the mapping is area preserving, there exists a generating function $g\left(x, y_{1}\right)$ which is defined by

$$
x_{1}=\frac{\partial g\left(x, y_{1}\right)}{\partial y_{1}}, \quad y=\frac{\partial g\left(x, y_{1}\right)}{\partial x} .
$$

Let $g\left(x, y_{1}\right)=x y_{1}+G\left(x, y_{1}\right)$. Simo proved [11] that a parabolic fixed point is surrounded by closed invariant curves if and only if $G\left(x, y_{1}\right)$ has a strict extremum there. To calculate the generating function for $(2.2)$, let us write

$$
\begin{aligned}
& x_{1}=x+p(x, y)+O\left(r^{h+1}\right)=x+p\left(x, y_{1}\right)+O\left(r^{h+i}\right)=\partial g / \partial y_{1} \\
& y=y_{1}-q(x, y)+O\left(r^{h+1}\right)=y_{1}-q\left(x, y_{1}\right)+O\left(r^{h+1}\right)=\partial g / \partial x
\end{aligned}
$$

By a calculation similar to $(2.8)$ we see here that

$$
g\left(x, y_{1}\right)=x y_{1}-\frac{x q-y_{1} p}{h+1}+O\left(r^{h+2}\right)
$$

and in this case the theorem follows from the result of Simo.

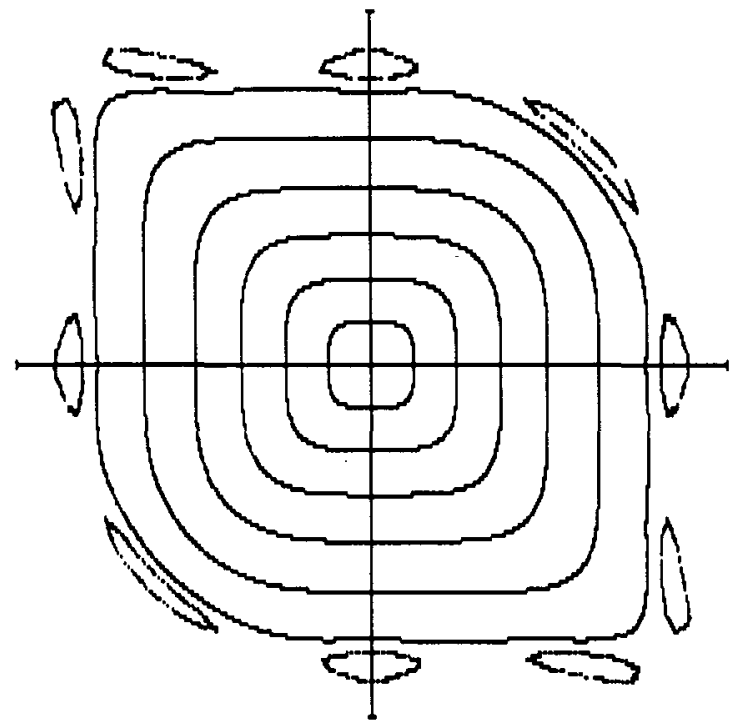

FIGURE 1. Invariant curves of the mapping (2.15). $-0.5<x, y<0.5$. 
Example 1. The conditions of Theorem 1 are fulfilled for the area-preserving mapping

$$
\begin{aligned}
& x_{1}=x+y^{2 n-1}, \\
& y_{1}=y-\left(x+y^{2 n-1}\right)^{2 n-1} .
\end{aligned}
$$

Here $h=2 n-1, H=x^{2 n}+y^{2 n}$. Some invariant curves for $2 n-1=3$ are shown in figure 1 . Each curve is generated by several hundred iterations of an initial point.

\section{Invariant curves near infinity}

In this section we discuss the mapping

$$
\begin{aligned}
& x_{1}=x+P(x, y), \\
& y_{1}=y+Q(x, y),
\end{aligned}
$$

where $P(x, y), Q(x, y) \rightarrow 0$ as $r^{2}=x^{2}+y^{2} \rightarrow \infty$, that is, when infinity is a parabolic fixed point. In particular we shall assume that (3.1) can be written near infinity as

$$
\begin{aligned}
& x_{1}=x+p(x, y)+\hat{p}(x, y), \\
& y_{1}=y+q(x, y)+\hat{q}(x, y),
\end{aligned}
$$

where $p(x, y), q(x, y)$ are real-analytic functions, homogeneous of negative degree $\boldsymbol{h}$, and $\hat{p}, \hat{q}$ are real-analytic functions such that

$$
\hat{p}(x, y), \hat{q}(x, y)=O\left(r^{h-1}\right)=O\left(r^{-(|h|+1)}\right) .
$$

There is a one-to-one correspondence between mappings with a fixed point at infinity and mappings with a similar fixed point at the origin. Let $T$ be a mapping which has a fixed point at infinity such that it is surrounded by closed invariant curves, and let $R$ be a one-to-one mapping which maps infinity to the origin. Then $R \circ T \circ R^{-1}$ has a fixed point at the origin, which is surrounded by invariant curves. Nevertheless, a parabolic fixed point at infinity deserves some separate discussion.

First, the area-preserving property does not have near infinity the same role that it has near a finite fixed point. As already mentioned, if an area-preserving mapping has a finite fixed point, then every closed curve which surrounds this fixed point intersects its image. This geometric reasoning fails for curves which surround a fixed point at infinity, since the area outside the closed curve and outside its image are both infinite. For example, the mapping

$$
\begin{aligned}
& x_{1}=\left(r^{2}+1\right)^{1 / 2} \cos \theta, \\
& y_{1}=\left(r^{2}+1\right)^{1 / 2} \sin \theta, \quad r^{2}=x^{2}+y^{2},
\end{aligned}
$$

is of type (3.2) and it has a fixed point at infinity, since for $r>1$

$$
\begin{aligned}
x_{1} & =\left(r^{2}+1\right)^{1 / 2} \cos \theta=r \cos \theta+\left(\left(1+r^{-2}\right)^{1 / 2}-1\right) r \cos \theta \\
& =x+\left(\frac{1}{2 r^{2}}-\frac{1}{8 r^{4}}+\cdots\right) x, \\
y_{i} & =y+\left(\frac{1}{2 r^{2}}-\frac{1}{8 r^{4}}+\cdots\right) y .
\end{aligned}
$$


On the other hand (3.4) can be written as

$$
r_{1}^{2}=r^{2}+1, \quad \theta_{1}=\theta \text {. }
$$

(3.5) is area preserving since $r_{1} d r_{1} d \theta_{1}=r d r d \theta$, but obviously no curve intersects its image under this mapping. Consequently, the existence of invariant curves near infinity is not implied by the area-preserving property alone, but it is related rather to the intersection property. One way to overcome this difficulty will be demonstrated at the end of $\S 4$.

Besides this remark, the existence of invariant curves of (3.1) around infinity can be shown as in Theorem 1

THEOREM 2. Given the real-analytic mapping (3.2) in a neighborhood of infinity and let $h \neq-1$. The fixed point at infinity is surrounded by closed invariant curves provided the following assumptions hold:

$$
\begin{gathered}
p_{x}+q_{y}=0 \\
x q(x, y)-y p(x, y) \neq 0 \quad \text { near infinity. }
\end{gathered}
$$

(c) Every closed curve sufficiently close to $x q-y p=c$ intersects its image curve.

The proof is similar to that of Theorem 1, except some minor changes due to $h \leq-2$. Here $x q-y p \rightarrow 0$ as $r \rightarrow \infty, q \hat{p}-p \hat{q}=O\left(r^{2 h-1}\right)$, and $H \geq A r^{h+1}$ implies $r \geq$ $B H^{1 /(h+1)}$. Thus (2.11)-(2.2) should be replaced by

$$
\begin{aligned}
\Delta \rho & =O\left(H^{-2 /(h+1)}\right)(q \hat{p}-p \hat{q})=O\left(H^{-2 /(h+1)} r^{2 h-1}\right) \\
& =O\left(H^{-2 /(h+1)} H^{(2 h-1) /(h+1)}\right)=O\left(H^{(2 h-3) /(h+1)}\right) \\
& =o\left(H^{2(h-1) /(h+1)}\right)=o\left(\rho^{2}\right) .
\end{aligned}
$$

The second reason which leads us to discuss the fixed point at infinity is the special case $h=-1$, which is strictly excluded from Theorem 2 . This case, where $p(x, y), q(x, y)$ are homogeneous of degree $h=-1$, is especially delicate and interesting and we shall discuss it now separately.

In the study of the case $h \neq-1$, we had introduced the integral curves $x q(x, y)-$ $y p(x, y)=c$ of the differential system (2.6)

$$
\begin{aligned}
& x^{\prime}=p(x, y), \\
& y^{\prime}=q(x, y), \quad h \neq-1,
\end{aligned}
$$

When $p(x, y)$ and $q(x, y)$ are homogeneous of degree $h=-1, x q-y p$ is not applicable any more, since it is identically constant. This follows from

$$
\begin{aligned}
& (x q-y p)_{x}=q+x q_{x}-y p_{x}=q+\left(x q_{x}+y q_{y}\right)=q+(-q)=0, \\
& (x q-y p)_{y}=0 .
\end{aligned}
$$

Hence we need a representation of the solutions of the system

$$
\begin{aligned}
& x^{\prime}=p(x, y), \\
& y^{\prime}=q(x, y), \quad h=-1 .
\end{aligned}
$$


LEMMA. Let

$$
\begin{gathered}
\lambda \stackrel{\text { def }}{=} x q(x, y)-y p(x, y), \\
\mu(\phi) \stackrel{\text { def }}{=} p(1, \operatorname{tg} \phi)+q(\cot \phi, 1) .
\end{gathered}
$$

(3.8) is a Hamiltonian system with

$$
H(x, y)=(\lambda / 2) \log \left(x^{2}+y^{2}\right)-\int^{\operatorname{arctg}(y / x)} \mu(\phi) d \phi .
$$

Its trajectories are closed curves if $\lambda \neq 0$ and

$$
\int_{0}^{2 \pi} \mu(\phi) d \phi=0
$$

Proof. (3.8) is explicitly solvable in polar coordinates $r=\left(x^{2}+y^{2}\right)^{1 / 2}, \phi=$ $\operatorname{arctg}(y / x)$ :

$$
\begin{gathered}
r^{\prime}(t)=\left(x x^{\prime}+y y^{\prime}\right) / r=(x p+y q) / r, \\
\phi^{\prime}(t)=\left(x y^{\prime}-y x^{\prime}\right) r^{-2}=(x q-y p) r^{-2} .
\end{gathered}
$$

Since $p(x, y)$ and $q(x, y)$ are homogeneous of degree -1 , the quantity

$$
x p(x, y)+y q(x, y)=p(1, y / x)+q(x / y, 1)
$$

depends only on $\phi=\operatorname{arctg}(y / x)$ and we denote it by

$$
\mu(\phi)=p(1, \operatorname{tg} \phi)+q(\cot \phi, 1) .
$$

by our previous remark $x q-y p$ is identically constant, say $\lambda$, and so (3.8) becomes $r^{\prime}=\mu(\phi) / r, \phi^{\prime}=\lambda r^{-2} \neq 0$. Its solutions are

$$
r(\phi)=r(0) \exp \left(\lambda^{-1} \int_{0}^{\phi} \mu(\phi) d \phi\right) .
$$

This is a closed curve if $\lambda \neq 0$ and (3.12) holds.

Differentiate now (3.11):

$$
H_{x}=\frac{\lambda}{2} \frac{2 x}{x^{2}+y^{2}}-\frac{-y}{x^{2}+y^{2}} \mu(\phi) .
$$

Substituting $\lambda$ and $\mu(\phi)$ from (3.10), the last quantity turns to be

$$
\frac{(x q-y p) x+y(x p+y q)}{x^{2}+y^{2}}=q .
$$

Similarly $H_{y}=-p$. Thus, $H$ is the Hamiltonian for (3.8).

The solutions of the differential system (3.8) are used to transform (3.1) into a twist mapping.

THEOREM 3. Given the mapping

$$
\begin{aligned}
& x_{1}=x+p(x, y)+\hat{p}(x, y) \\
& y_{1}=y+q(x, y)+\hat{q}(x, y),
\end{aligned}
$$


near infinity, where $p(x, y), q(x, y)$ are real-analytic functions, homogeneous of degree -1 , and $\hat{p}, \hat{q}$ are real-analytic functions such that

$$
\hat{p}(x, y), \hat{q}(x, y)=O\left(r^{-2}\right) .
$$

The fixed point at infinity is surrounded by closed invariant curves provided the following assumptions hold:

(a)

$$
p_{x}+q_{y}=0
$$

and the constant $x q-y p$ is nonzero.

(b) The function

$$
\mu(\phi)=p(1, \operatorname{tg} \phi)+q(\cot \phi, 1)
$$

is integrable and satisfies

$$
\int_{0}^{2 \pi} \mu(\phi) d \phi=0 .
$$

(c) Every closed curve sufficiently close to $r=c \exp \left(\lambda^{-1} \int_{0}^{\phi} \mu(t) d t\right)$ intersects its image curve.

Proof. Let $\left.G(x, y) \equiv \exp (H)=r^{\lambda} \exp \left(-\int \mu(\phi) d \phi\right)\right)$ and

$$
\begin{aligned}
& \theta=\int_{0}^{\operatorname{arctg}(y / x)} G(\cos \phi, \sin \phi)^{-2 / \lambda} d \phi, \\
& \rho=G(x, y)^{-2 / \lambda}=r^{-2} \exp \left(2 \int \mu(\phi) / \lambda d \phi\right) .
\end{aligned}
$$

then, by using that $\rho=G^{-2 / \lambda}$ is a homogeneous function of degree -2 ,

$$
\begin{aligned}
\theta_{1}-\theta=\Delta \theta & =\frac{x \Delta y-y \Delta x}{r^{2}}(1+o(1)) G(\cos \phi, \sin \phi)^{-2 / \lambda} \\
& =(x Q-y P) r^{-2} G^{-2 / \lambda}(x / r, y / r)(1+o(1)) \\
& =\left(\lambda+o\left(r^{-1}\right)\right) G^{-2 / \lambda}(x, y)(1+o(1)) \\
& =\lambda \rho(1+o(1)) . \\
\rho_{1}-\rho=\Delta \rho & =(-2 / \lambda) G^{-2 / \lambda-1}\left[G_{x} \Delta x+G_{y} \Delta y+\frac{1}{2} G_{x x} \Delta x^{2}+\cdots\right] \\
& =(-2 /) G^{-2 / \lambda}\left[\left(G_{x} / G\right) P+\left(G_{y} / G\right) Q+\left(G_{x x} / G\right) P^{2}+\cdots\right] .
\end{aligned}
$$

Now $G_{x} / G=q, G_{x x} / G=q_{x}+q^{2}, G_{x y} / G=q_{y}-q p$, etc, so

$$
\begin{aligned}
& =(-2 / \lambda) \rho\left[q P-p Q+\frac{1}{2}\left(q_{x}+q^{2}\right) P^{2}+\cdots\right] \\
& =(-2 / \lambda) \rho\left[q \hat{p}-p \hat{q}+O\left(r^{-4}\right)\right] \\
& =\rho O\left(r^{-3}\right)=O\left(\rho^{5 / 2}\right) .
\end{aligned}
$$

Now, the doubly connected domain $\infty>r \geq A \exp \left(\int_{0}^{\phi} \mu(t) / \lambda d t\right)$ is mapped onto $0<\rho \leq A^{-2}$. Thus, the mapping (3.1) had been transformed into a twist mapping

$$
\begin{aligned}
& \theta_{1}=\theta+\lambda \rho+f(\rho, \theta), \\
& \rho_{1}=\rho+g(\rho, \theta),
\end{aligned}
$$


on the ring $0<\rho \leq A^{-2}$, where $f(\rho, \theta), g(\rho, \theta)$ are real analytic, periodic in $\theta$ and $f=o(\rho), g=O\left(\rho^{5 / 2}\right)$ as $\rho \rightarrow 0$. If we replace $\rho$ by $A^{-2} \rho,(3.16)$ is transformed into

$$
\begin{aligned}
& \theta_{1}=\theta+\lambda A^{-2} \rho+\tilde{f}(\rho, \theta), \\
& \rho_{1}=\rho+\tilde{\mathbf{g}}(\rho, \theta),
\end{aligned}
$$

on $0<\rho \leq 1$, where $\tilde{f}(\rho, \theta)=f\left(A^{-2} \rho, \theta\right)=o\left(A^{-2}\right), \tilde{g}(\rho, \theta)=A^{2} g\left(A^{-2} \rho, \theta\right)=O\left(A^{-3}\right)$. Here $(|\tilde{f}|+|\tilde{g}|) / \lambda A^{-2} \rightarrow 0$ as $A \rightarrow \infty$, as required in the theory of Moser.

The condition $\lambda \neq 0$ in Theorem 3 is essential. For example, the mapping

$$
\begin{aligned}
& x_{1}=x+x y / r^{3}, \\
& y_{1}=y+y^{2} / r^{3},
\end{aligned}
$$

which may be written as

$$
r_{1}=r+y / r^{2}, \quad \phi_{1}=\phi,
$$

satisfies all the assumptions of Theorem 3 except that $x q-y p \equiv 0$, and it has obviously no closed invariant curves.

\section{An interesting example}

In [1] there appeared the following problem (6439): prove that a sequence $\left\{a_{n}\right\}$ of real numbers satisfying $a_{n+1}=\left|a_{n}\right|-a_{n-1}$ is periodic of period 9. An elementary solution is based on the observation that if, for example, two consecutive terms of the sequence, say $a, b$, are nonpositive, then the first eleven terms of the sequence are

$$
a, b,-a-b,-a-2 b,-b, a+b,-a,-2 a-b,-a-b, a, b, \ldots
$$

T. Sheil-Small asked one of the authors whether a sequence of complex numbers $\left\{z_{n}\right\}$ satisfying

$$
z_{n+1}=\left|z_{n}\right|-z_{n-1}
$$

is bounded? We shall answer this question in affirmative, using methods similar to our previous results. This example reveals a delicate structure of a dynamical system, surpassing in its importance the original question.

Since by (4.2) $\operatorname{Im}\left\{z_{n+1}\right\}=-\operatorname{Im}\left\{z_{n-1}\right\}$, we let $a=\operatorname{Im}\left\{z_{0}\right\}, b=\operatorname{Im}\left\{z_{1}\right\}, x_{n}=\operatorname{Re}\left\{z_{2 n}\right\}$, $y_{n}=\operatorname{Re}\left\{z_{2 n+1}\right\}, n=0,1, \ldots$ By this notation (4.2) may be written as

$$
\begin{aligned}
& x_{n+1}=\left(y_{n}^{2}+b^{2}\right)^{1 / 2}-x_{n} \\
& y_{n+1}=\left(x_{n+1}^{2}+a^{2}\right)^{1 / 2}-y_{n} .
\end{aligned}
$$

Consider the mapping

$$
T: \begin{aligned}
& x_{1}=\left(y^{2}+b^{2}\right)^{1 / 2}-x \\
& y_{1}=\left(x_{1}^{2}+a^{2}\right)^{1 / 2}-y .
\end{aligned}
$$

For the sake of simplicity we shall always assume that $a, b \neq 0 . T$ is a real analytic, area preserving, one-to-one mapping in the whole $(x, y)$ plane. Our aim is to prove that its 9 th power, $S=T^{9}$, has a parabolic fixed point at infinity which is surrounded 
by invariant curves. This will imply, among other things, that every sequence satisfying (4.2) is bounded.

The forthcoming proof is based on the following heuristic considerations:

If both $|y|$ and $|y|-x$ are large, then (4.4) approximates the piecewise linear mapping

$$
T_{0}: \begin{aligned}
& x_{1}=|y|-x, \\
& y_{1}=|| y|-x|-y,
\end{aligned}
$$

which corresponds to the real version of (4.2). Motivated by (4.1), we divide the plane into 9 open cones

$$
\begin{array}{ccc}
K_{0}=\{x<0, y<0\}, & K_{1}=\{x<y<2 x\}, & K_{2}=\{0<x<-y\}, \\
K_{3}=\{0<x<2 y\}, & K_{4}=\{-x<y<0\}, & K_{5}=\{0<-x<y\}, \\
K_{6}=\{0<2 y<x\}, & K_{7}=\{0<y<-x), & K_{8}=\left\{\frac{1}{2} x<y<x\right\},
\end{array}
$$

separated by rays through the origin. (See figure 2.) It is easy to see that $T_{0}$ is linear on each $K_{i}$ and maps it in a one to one manner onto $K_{i+1}, i(\bmod 9)$. By the solution of problem [1] it is obvious that $T_{0}^{9}=I$.

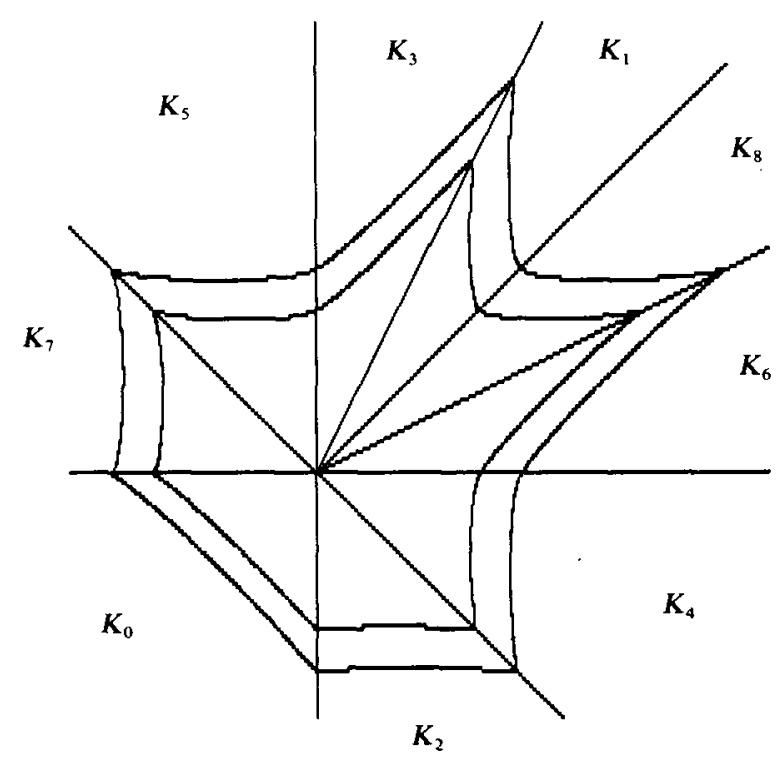

Figure 2. The cones $K_{0}, \ldots, K_{8}$ for the mapping (4.4) with $a=2, b=0.3$ and two invariant curves generated by iterations of initial points $(40,0),(50,0)$.

Next we see how does $S=T^{9}$ act on each cone $K_{i}$, close to infinity but away from the boundary of the cone. We begin, for example, with $K_{0}=\{x, y<0\}$ but restrict ourselves to a narrower cone

$$
\tilde{K}_{0}=\{\pi+\alpha<\arg (y / x)<3 \pi / 2-\alpha\}, \quad \alpha>0 .
$$


On this restricted cone $T_{0}$ approximates $T$ near infinity up to $O\left(r^{-1}\right)$. Indeed, when $r^{2}=x^{2}+y^{2} \rightarrow \infty$ in $\tilde{K}_{0}$, then $-y>0$ is large and so

$$
\begin{aligned}
x_{1} & =\left(y^{2}+b^{2}\right)^{1 / 2}-x=(-y)\left(1+b^{2} / 2 y^{2}+O\left(r^{-4}\right)\right)-x \\
& =-x-y-b^{2} / 2 y+O\left(r^{-3}\right) .
\end{aligned}
$$

$-x-y>0$ is large, too, so

$$
\begin{aligned}
y_{1} & =\left(\left[x+y+b^{2} / 2 y+O\left(r^{-4}\right)\right]^{2}+a^{2}\right)^{1 / 2}-y \\
& =(-x-y)\left(1+(x+y)^{-2}\left((x+y) b^{2} / 2 y+a^{2}\right)+O\left(r^{-4}\right)\right)-y \\
& =-x-2 y+\frac{1}{2}\left(b^{2} / 2 y+a^{2} /(x+y)\right)+O\left(r^{-3}\right) .
\end{aligned}
$$

For $(x, y) \in \tilde{K}_{0},-x-2 y$ is large and $b^{2} / 2 y+a^{2} /(x+y)=O\left(r^{-1}\right)$, so we may calculate the following iterations according to the same considerations and get

$$
\begin{aligned}
& x_{2}=-y+\frac{1}{2}\left(-a^{2} /(x+y)-b^{2} /(x+2 y)\right)+O\left(r^{-3}\right), \\
& y_{2}=x+y+\frac{1}{2}\left(-b^{2} /(x+2 y)+\left(-a^{2}+b^{2}\right) / y\right)+O\left(r^{-3}\right) .
\end{aligned}
$$

After the ninth iteration we obtain

$$
\begin{aligned}
& x_{9}=x-\frac{1}{2}\left(a^{2}+b^{2}\right)\left(\frac{2}{x+2 y}+\frac{1}{x+y}+\frac{1}{2 x+y}\right)+O\left(r^{-3}\right), \\
& y_{9}=y+\frac{1}{2}\left(a^{2}+b^{2}\right)\left(\frac{1}{x+2 y}+\frac{1}{x+y}+\frac{2}{2 x+y}\right)+O\left(r^{-3}\right), \quad(x, y) \in \tilde{K}_{0} .
\end{aligned}
$$

Similar calculations in the other cones, away from their boundaries, lead us to conclude that $S=T^{9}$ may be written in each restricted cone $\tilde{K}_{i}$ as

$$
S=T^{9}: \begin{aligned}
& x_{1}=x+p(x, y)+O\left(r^{-3}\right), \\
& y_{1}=y+q(x, y)+O\left(r^{-3}\right),
\end{aligned}
$$

where $p, q$ are homogeneous of degree -1 . In particular

$$
T^{9}-I=\left(\begin{array}{l}
p(x, y) \\
q(x, y)
\end{array}\right)\left(1+O\left(r^{-2}\right)\right) \quad \text { on } \cup \tilde{K}_{i} .
$$

Let us put this into the identity $T^{9}-I=T\left(T^{9}-I\right) T^{-1}$; since on every restricted cone $\tilde{K}_{i}, T(x, y)=T_{0}(x, y)\left(1+O\left(r^{-1}\right)\right)$, it follows that

$$
\left(\begin{array}{l}
p(x, y) \\
q(x, y)
\end{array}\right)=T_{0} \circ\left(\begin{array}{l}
p(,) \\
q(,)
\end{array}\right) \circ T_{0}^{-1}(x, y)
$$

Thus, the analytic expressions for $p(x, y), q(x, y)$ in the various $\tilde{K}_{i}-s$ are related by

$$
\left.\left(\begin{array}{l}
p(x, y) \\
q(x, y)
\end{array}\right)\right|_{\tilde{\kappa}_{i}}=\left.T_{0}^{i} \circ\left(\begin{array}{l}
p(,) \\
q(,)
\end{array}\right)\right|_{\tilde{\kappa}_{0}} \circ T_{0}^{-i}(x, y), \quad(x, y) \in \tilde{K}_{i} .
$$

The functions $p, q$ in (4.7) are analytic in each $\tilde{K}_{i}, i=0, \ldots, 8$, separately, but discontinuous on 9 rays. On the other hand, the mapping $S=T^{9}$ is obviously real analytic in the whole $(x, y)$ plane. Hence we are faced with the difficulty to represent $S$ by an expression similar to (4.7) and valid in the whole plane and to decide how well the piecewise analytic $p, q$ which we found above, represent our mapping. 
To understand the role of $p, q$ that were obtained in $\tilde{K}_{i}$, we continue them analytically to the whole open cones $K_{i}$. That is, let $p, q$ be defined by (4.6) on the whole $K_{0}=\{x, y<0\}$ and by the identity (4.8) on $K_{i}, i=1, \ldots, 8$. Now we consider in the whole plane the autonomous system of differential equations

$$
\begin{aligned}
& x^{\prime}=p(x, y), \\
& y^{\prime}=q(x, y),
\end{aligned}
$$

where $p, q$ are discontinuous on 9 rays. In $K_{0}$, for example, the solutions of

$$
\begin{aligned}
& x^{\prime}=-\frac{1}{2}\left(a^{2}+b^{2}\right)\left(\frac{2}{x+2 y}+\frac{1}{x+y}+\frac{1}{2 x+y}\right), \\
& y^{\prime}=\frac{1}{2}\left(a^{2}+b^{2}\right)\left(\frac{1}{x+2 y}+\frac{1}{x+y}+\frac{2}{2 x+y}\right),
\end{aligned}
$$

are

$$
F(x, y) \stackrel{\text { def }}{\equiv}[(x+2 y)(x+y)(2 x+y)]^{1 / 2\left(a^{2}+b^{2}\right)} \times \text { constant; }
$$

also

$$
F_{x} / F=q, \quad F_{y} / F=-p .
$$

In each other $K_{i}$ the solutions are given by

$$
F \circ T^{-i}(x, y)=\text { constant. }
$$

The identity $T_{0}^{9}=I$ shows that these 9 families of curves form closed trajectories and all solutions of (4.9) are periodic.

At this point it is evident that the assumptions of Theorem 3 are too restrictive to be applicable for the mapping (4.4). The following theorem will apply to the mapping (4.4), too.

THEOREM 4. Given the real-analytic mapping

$$
\begin{aligned}
& x_{1}=x+P(x, y), \\
& y_{1}=y+Q(x, y),
\end{aligned}
$$

which may be written also as

$$
\begin{aligned}
& x_{1}=x+p(x, y)+\hat{p}(x, y), \\
& y_{1}=y+q(x, y)+\hat{q}(x, y),
\end{aligned}
$$

where $p, q, \hat{p}, \hat{q}$ satisfy the following assumptions:

(a) $p, q$ are homogeneous of degree -1 and differentiable, except on a finite number of rays.

(b) Whenever $p, q$ are differentiable, they satisfy

$$
p_{x}+q_{y}=0
$$

and the constant $x q-y p$ is nonzero. 
(c) The solutions of the system of autonomous differential equations

$$
\begin{aligned}
& x^{\prime}=p(x, y), \\
& y^{\prime}=q(x, y),
\end{aligned}
$$

are periodic.

The functions $\hat{p}, \hat{q}$ satisfy the following smallness conditions:

(d) $\int_{t_{1}}^{t_{2}} \hat{p}(A \cos t, A \sin t) d t=o\left(A^{-1}\right), \int_{t_{1}}^{t_{2}} \hat{q}(A \cos t, A \sin t) d t=o\left(A^{-1}\right), A \rightarrow \infty$.

(e) $x \hat{q}-y \hat{p}=o(1)$ near infinity.

(f) $\lim P(x, y) / Q(x, y)$ exists when $(x, y)$ tends to infinity along any ray through $(0,0)$.

\section{Finally,}

(g) Every closed curve which surrounds infinity intersects its image under the mapping (4.4).

Then the fixed point of (4.4) at infinity is surrounded by closed invariant curves.

Note that if $\hat{p}, \hat{q}=o\left(r^{-2}\right)$, then (d), (e), (f) are automatically fulfilled.

Proof. We have already seen that if $p, q$ are homogeneous of degree -1 and $p_{x}+q_{y}=0$, then $x q-y p$ is identically constant. Since $x Q-y P$ is real analytic in the whole plane and $x \hat{q}-y \hat{p}=o(1)$, it is the same constant in domains which are separated by rays where $p, q$ are not differentiable. So let

$$
\lambda \stackrel{\text { def }}{\equiv} x \boldsymbol{q}-\boldsymbol{y p}
$$

and suppose $\lambda \neq 0$. As $x p+y q$ depends only on $y / x=\tan (t)$, we denote again

$$
\mu(t) \stackrel{\text { def }}{=} x p(x, y)+y q(x, y)=p(y / x, 1)+q(1, x / y) \text {. }
$$

It was seen in (3.13) that the solutions of the system (4.12) are

$$
r=c \exp \left(-\int_{0}^{\phi} \mu(t) / \lambda d t\right)
$$

and they are periodic if and only if

$$
\int_{0}^{2 \pi} \mu(t) / \lambda d t=0 .
$$

Also observe that

$$
\mu(t) / \lambda=(x p+y q) /(x q-y p) .
$$

Motivated by (4.15)-(4.17), we shall suggest new variables such that (4.11) will become an analytic twist mapping near infinity. Let

$$
M=\max _{0 \leq \phi \leq 2 \pi}\left(\exp \int_{0}^{\phi} \mu(t) / \lambda d t\right) .
$$

For a positive number $A$, let $\mathscr{D}_{A}$ be the external domain

$$
\mathscr{D}_{A}: \quad r \geq A / M\left(\exp \int_{0}^{\phi} \mu(t) / \lambda d t\right) .
$$

The circle $r=A$ is contained in $\mathscr{D}_{A}$.

Because of (4.15)-(4.17), we begin our proof by estimating

$$
\int_{0}^{2 \pi} \frac{\xi P(\xi, \eta)+\eta Q(\xi, \eta)}{\eta Q(\xi, \eta)-\eta P(\xi, \eta)} d t
$$


where $\xi=A \cos (t), \eta=A \sin (t)$, when $A \rightarrow \infty$. For short we let $P=P(\xi, \eta), p=$ $p(\xi, \eta)$, etc. Since $x q-y p \equiv \lambda, x Q-y P=(x q-y p)+(x \hat{q}-y \hat{p})=\lambda+o(1)$, we have

$$
\frac{\xi P+\eta Q}{\xi Q-\eta P}-\frac{\xi p+\eta q}{\xi q-\eta p}=\frac{\left(\xi^{2}+\eta^{2}\right)(q P-p Q)}{(\xi Q-\eta P)(\xi q-\eta p)}=\frac{A^{2}(q \hat{p}-p \hat{q})}{(\lambda+o(1)) \lambda}
$$

Integrating this, we obtain by identity (4.17) and assumptions (a), (d),

$$
\int_{t_{1}}^{t_{2}} \frac{\xi P+\eta Q}{\xi Q-\eta P} d t-\int_{t_{1}}^{t_{2}} \mu(t) / \lambda d t=O\left(A^{2}\right) \int_{t_{1}}^{t_{2}}(p \hat{q}-q \hat{p}) d t=o(1) .
$$

In particular, we obtain on $[0,2 \pi]$

$$
\int_{0}^{2 \pi} \frac{\xi P+\eta Q}{\xi Q-\eta P} d t=o(1)
$$

Therefore the number $\varepsilon(A)$ defined by

$$
2 \pi \varepsilon(A) \stackrel{\text { def }}{=} \int_{0}^{2 \pi} \frac{\xi P+\eta Q}{\xi Q-\eta P} d t
$$

tends to 0 as $A \rightarrow \infty$. Moreover,

$$
\int_{0}^{2 \pi}\left[\frac{\xi P+\eta Q}{\xi Q-\eta P}-\varepsilon(A)\right] d t=0
$$

and

$$
\int_{0}^{t}\left[\frac{\xi P+\eta Q}{\xi Q-\eta P}-\varepsilon(A)\right] d t
$$

is a real analytic, periodic function of $t$.

To this end we define new variables $\rho, \theta$ as

$$
\begin{aligned}
& \rho=\left(x^{2}+y^{2}\right)^{-1} \exp \left(2 \int_{0}^{\operatorname{arctg}(y / x)}\left[\frac{\xi P+\eta Q}{\xi Q-\eta P}-\varepsilon(A)\right] d t\right), \\
& \theta=\int_{0}^{\operatorname{arctg}(y / x)} \exp \left(2 \int_{0}^{\phi}\left[\frac{\xi P+\eta Q}{\xi Q-\eta P}-\varepsilon(A)\right] d t\right) d \phi,
\end{aligned}
$$

where the value of arctg is that of the argument which corresponds to the point $(x, y)$ as it surrounds the origin.

$\rho, \theta$ are real-analytic functions of $(x, y) . \rho$ decreases along any ray through $(0,0)$ and $\theta$ increases with $\arg (y / x)$, hence the correspondence $(\rho, \theta) \leftrightarrow(x, y)$ is locally one to one. When $(x, y)$ surrounds $(0,0)$ and returns to its original location, $\rho$ admits, by (4.19), the same value; in the same time $\theta$ increases, according to (4.18), by

$$
\begin{aligned}
\omega=\omega(A) & =\int_{0}^{2 \pi} \exp \left(2 \int_{0}^{\phi}\left[\frac{\xi P+\eta Q}{\xi Q-\eta P}-\varepsilon(A)\right] d t\right) d \phi \\
& =\int_{0}^{2 \pi} \exp \left(2 \int_{0}^{\phi} \mu(t) / \lambda d t\right) d \phi(1+o(1))
\end{aligned}
$$

Hence our change of variables is even globally one-to-one. 
By the estimate (4.18),

$$
\begin{aligned}
\rho & =\left(x^{2}+y^{2}\right)^{-1} \exp \left(2 \int_{0}^{\operatorname{arctg}(y / x)}\left[\frac{\xi P+\eta Q}{\xi Q-\eta P}-\varepsilon(A)\right] d t\right) \\
& =\left(x^{2}+y^{2}\right)^{-1} \exp \left(2 \int_{0}^{\operatorname{arctg}(y / x)} \mu(t) / \lambda d t\right)(1+o(1)) .
\end{aligned}
$$

As already mentioned, the last two expressions keep their original values after surrounding the origin, hence also the $o(1)$ depends periodically on $\arg (y / x)$. Combining the last estimate with the definition of the domain $\mathscr{D}_{A}$, we see that $\mathscr{D}_{A}$ is mapped onto

$$
0<\rho \leq(M / A)^{2}(1+o(1)) .
$$

Now we describe the action of our mapping (4.4) in terms of the coordinates $\rho, \theta$.

$$
\begin{aligned}
\theta_{1}-\theta & =\Delta \theta \\
& =\frac{x \Delta y-y \Delta x}{r^{2}} \exp \left(2 \int_{0}^{\operatorname{arctg}(y / x)}\left[\frac{\xi P+\eta Q}{\xi Q-\eta P}-\varepsilon(A)\right] d t\right)(1+o(1)) \\
& =(x Q-y P) \rho(1+o(1)) .
\end{aligned}
$$

But $x Q-y P=\lambda+o(1)$, so

$$
\theta_{1}-\theta=\lambda \rho+o(\rho)
$$

The change of $\rho$ may be calculated by

$$
\begin{gathered}
\rho_{1}-\rho=\Delta \rho \cong \rho_{x} \Delta x+\rho_{y} \Delta y \\
=\rho_{x} P+\rho_{x} Q \\
\rho_{x}=\exp \left(2 \int_{0}^{\operatorname{arctg}(y / x)}\left[\frac{\xi P+\eta Q}{\xi Q-\eta P}-\varepsilon(A)\right] d t\right) \\
\times\left(-2 x\left(x^{2}+y^{2}\right)^{-2}-2 y\left(x^{2}+y^{2}\right)^{-2}\left[\frac{\xi P+\eta Q}{\xi Q-\eta P}-\varepsilon(A)\right]_{t=\operatorname{arctg}(y / x)}\right) \\
=-2\left(x^{2}+y^{2}\right)^{-1} \rho\left[x+y \frac{x P(x, y)+y Q(x, y)}{x Q(x, y)-y P(x, y)}+y\left(-\varepsilon(A)+\left.\frac{x P+y Q}{x Q-y P}\right|_{(x, y)} ^{(\xi, \eta)}\right)\right],
\end{gathered}
$$

where $(\xi, \eta)$ denotes, as before, $(A \cos t, A \sin t)$ and $t=\operatorname{arctg}(y / x)$. But

$$
x+y \frac{x P+y Q}{x Q-y P}=\frac{\left(x^{2}+y^{2}\right) Q}{x Q-y P}
$$

therefore

$$
\rho_{x} P=-2 \rho \frac{Q P}{x Q-y P}+2 \rho\left(x^{2}+y^{2}\right)^{-1}(-y P)\left(-\varepsilon(A)+(x P+y Q) /\left.(x Q-y P)\right|_{(x, y)} ^{(\xi, \eta)}\right) .
$$

A similar calculation shows that

$$
\rho_{y} Q=2 \rho \frac{P Q}{x Q-y P}+2 \rho\left(x^{2}+y^{2}\right)^{-1}(x Q)\left(-\varepsilon(A)+(x P+y Q) /\left.(x Q-y P)\right|_{(x, y)} ^{(\xi, \eta)}\right),
$$

and

$$
\rho_{x} P+\rho_{y} Q=2 \rho\left(x^{2}+y^{2}\right)^{-1}(x Q-y P)\left(-\varepsilon(A)+(x P+y Q) /\left.(x Q-y P)\right|_{(x, y)} ^{(\xi, \eta)}\right) .
$$


By the definition of $\rho,\left(x^{2}+y^{2}\right)^{-1}=O(\rho)$; also $x Q-y P=\lambda+o(1)$, so

$$
\Delta \rho=O\left(\rho^{2}\right)\left(-\varepsilon(A)+(x P+y Q) /\left.(x Q-y P)\right|_{(x, y)} ^{(\xi, \eta)}\right)
$$

and all we have to do is to estimate $(x P+y Q) /\left.(x Q-y P)\right|_{(x, y)} ^{(\xi, \eta)}$.

According to (f), $(x p+y Q) /(x Q-y P)=(P / Q+y / x) /(1-(P / Q)(y / x))$ tends to a limit when $(x, y)$ tends to infinity along any ray $y / x=$ constant. The points $(x, y)$, $(\xi, \eta)$ are located on the same ray and in $\mathscr{D}_{A}$, So when $A$ is sufficiently large, the difference $(x P+y Q) /\left.(x Q-y P)\right|_{(x, y)} ^{(\xi, \eta)}$ is arbitrary small, that is $o(1)$, and we have proved that $\Delta \rho=o\left(\rho^{2}\right)$. Thus, the mapping (4.11) had been transformed into a twist mapping

$$
\begin{aligned}
& \theta_{1}=\theta+\lambda \rho+f(\rho, \theta), \\
& \rho_{1}=\rho+g(\rho, \theta),
\end{aligned}
$$

on the ring $0<\rho \leq(M / A)^{2}(1+o(1))$, where $f(\rho, \theta), g(\rho, \theta)$ are real analytic, periodic in $\theta$ and $f=o(\rho), g=o\left(\rho^{2}\right)$ as $\rho \rightarrow 0$. If we replace $\rho$ by $A^{-2} \rho,(4.23)$ is transformed into

$$
\begin{aligned}
& \theta_{1}=\theta+\lambda A^{-2} \rho+\tilde{f}(\rho, \theta), \\
& \rho_{1}=\rho+\tilde{g}(\rho, \theta),
\end{aligned}
$$

on $0<\rho \leq 1+o(1)$, where $\tilde{f}(\rho, \theta)=f\left(A^{-2} \rho, \theta\right)=o\left(A^{-2}\right), \tilde{g}(\rho, \theta)=A^{2} g\left(A^{-2} \rho, \theta\right)=$ $o\left(A^{-2}\right)$. If we denote $\gamma=\lambda A^{-2}$, then $(|\tilde{f}|+|\tilde{g}|) / \gamma \rightarrow 0$ as $A \rightarrow \infty$, and all the requirements of the theory of Moser are satisfied for sufficiently large values of $A$.

Now we return to the mapping (4.4) and prove that $S=T^{9}$ satisfies all the requirements of Theorem 4 . In our preliminary calculations we obtained (4.7) away from the boundaries of the cones $K_{i}$. Next we shall obtain identities which are valid also on the boundaries. For example, $K_{0}$ and $K_{2}$ are separated by the ray $x=0$, $y<0$. So we consider the cone $K_{02}=\left\{\pi+\alpha<\arg (y / x)<\frac{7}{4} \pi-\alpha\right\}, \alpha>0$, which contains most of $K_{0} \cup K_{2} \cup\{x=0, y<0\}$.

On $K_{02}\left(x_{1}, y_{1}\right),\left(x_{2}, y_{2}\right)$ are calculated as in $\tilde{K}_{0}$ and

$$
x_{3}=-x+\frac{1}{2}\left(2 b^{2} /(x+2 y)+\left(a^{2}-b^{2}\right) / y+\left(a^{2}-b^{2}\right) /(x+y)\right)+O\left(r^{-3}\right) .
$$

Next

$$
y_{3}=\left(\left(-x+\rho_{1}\right)^{2}+a^{2}\right)^{1 / 2}-y_{2},
$$

where $\rho_{1}$ denotes the $O\left(r^{-1}\right)$ terms in $x_{3}$. However, in $K_{02}$ the term $-x$ may be large or small relative to $\rho_{1}$ and $a$, so we define

$$
\zeta_{1} \stackrel{\text { def }}{\equiv}\left(\left(-x+\rho_{1}\right)^{2}+a^{2}\right)^{1 / 2}
$$

Obviously $\zeta_{i}=|x|+O(1)$. Using this notation and (4.5),

$$
y_{3}=\zeta_{1}-y_{2}=-x-y+\zeta_{1}+\frac{1}{2}\left(b^{2} /(x+2 y)+\left(a^{2}-b^{2}\right) / y\right)+O\left(r^{-3}\right) \text {. }
$$


In $x_{4}$ we meet the term $b^{2} /\left(-x-y+\zeta_{1}\right)$. It will be replaced by $-b^{2} /(x+y-|x|)+$ $O\left(r^{-2}\right)$ to obtain

$$
x_{4}=-y+\zeta_{1}+\frac{1}{2}\left(-b^{2} /(x+2 y)+\left(-a^{2}+b^{2}\right) /(x+y)-b^{2} /(x+y-|x|)\right)+O\left(r^{-2}\right) .
$$

In the next calculation we replace $1 /\left(-y+\zeta_{1}\right)$ by $1 /(-y+|x|)+O\left(r^{-2}\right)$ :

$$
\begin{aligned}
& y_{4}=x+\frac{1}{2}(-2 b^{2} /(x+2 y)+\left(-a^{2}+b^{2}\right) /(x+y)-b^{2} /(x+y-|x|)+a^{2} /(-y+|x|) \\
&\left.+\left(-a^{2}+b^{2}\right) / y\right)+O\left(r^{-2}\right),
\end{aligned}
$$

Here, again, $x$ may be small or large, so we define

$$
\zeta_{2} \stackrel{\text { def }}{=}\left(\left(x+\rho_{2}\right)^{2}+b^{2}\right)^{1 / 2}, \quad \zeta_{2}=|x|+O(1) .
$$

where $\rho_{2}$ denotes the $O\left(r^{-1}\right)$ terms in $y_{4}$. Therefore

$$
\begin{aligned}
x_{5} & =\zeta_{2}-x_{4} \\
& =y+\zeta_{2}-\zeta_{1}+\frac{1}{2}\left(b^{2} /(x+2 y)+\left(a^{2}-b^{2}\right) /(x+y)+b^{2} /(x+y-|x|)\right)+O\left(r^{-2}\right) .
\end{aligned}
$$

In the next step we replace $1 /\left(y+\zeta_{2}-\zeta_{1}\right)$ by $1 / y+O\left(r^{-2}\right)$, and continue according to the same scheme until

$$
y_{7}=-x+\frac{1}{2}\left(2\left(a^{2}+b^{2}\right) /(x+2 y)-a^{2} /(-y+|x|)+b^{2} /(x+y-|x|)\right)+O\left(r^{-2}\right)
$$

Now we put

$$
\zeta_{3}=\left(\left(-x+\rho_{3}\right)^{2}+b^{2}\right)^{1 / 2}, \quad \zeta_{3}=|x|+O(1) .
$$

and continue the iterations until we encounter

$$
x_{9}=x+\frac{\left(a^{2}+b^{2}\right)}{2}(-2 /(x+2 y)-1 /(-y+|x|)-1 /(x+y-|x|))+O\left(r^{-2}\right) .
$$

Finally let

$$
\zeta_{4}=\left(\left(x+\rho_{4}\right)^{2}+a^{2}\right)^{1 / 2}, \quad \zeta_{4}=|x|+O(1) .
$$

Then

$$
\begin{aligned}
y_{9} & =\zeta_{4}-y_{8} \\
& =y+\zeta_{4}-\zeta_{3}+\zeta_{2}-\zeta_{1}+\frac{1}{2}\left(a^{2}+b^{2}\right)[1 /(x+2 y)+1 /(x+y-|x|))+O\left(r^{-2}\right) .
\end{aligned}
$$

To get a better idea about $T^{9}$, we must estimate $\zeta_{4}-\zeta_{3}+\zeta_{2}-\zeta_{1}$. Since $\rho_{i}=O\left(r^{-1}\right)$,

$$
\begin{aligned}
& \zeta_{4}-\zeta_{1}=\left(\left(x+\rho_{4}\right)^{2}+a^{2}\right)^{1 / 2}-\left(\left(-x+\rho_{1}\right)^{2}+a^{2}\right)^{1 / 2}=x\left(\rho_{4}+\rho_{1}\right) /\left(x^{2}+a^{2}\right)^{1 / 2}+O\left(r^{-2}\right), \\
& \zeta_{2}-\zeta_{3}=x\left(\rho_{3}+\rho_{2}\right) /\left(x^{2}+b^{2}\right)^{1 / 2}+O\left(r^{-2}\right),
\end{aligned}
$$

and

$$
\begin{aligned}
\zeta_{4}-\zeta_{3} & +\zeta_{2}-\zeta_{1} \\
= & \left(x /\left(x^{2}+a^{2}\right)^{1 / 2}-x /\left(x^{2}+b^{2}\right)^{1 / 2}\right)\left(\rho_{4}+\rho_{1}\right)+x /\left(x^{2}+b^{2}\right)^{1 / 2}\left(\rho_{4}+\rho_{3}+\rho_{2}+\rho_{1}\right) \\
& +O\left(r^{-2}\right)
\end{aligned}
$$

By (4.25)-(4.28)

$$
\rho_{4}+\rho_{3}+\rho_{2}+\rho_{1}=\frac{1}{2}\left(a^{2}+b^{2}\right)(-1 /(-y+|x|)-1 /(x+y-|x|))+O\left(r^{-2}\right) .
$$


So finally we write $S=T^{9}$ on $K_{\alpha 2}$ as

$$
\begin{aligned}
x_{9}= & x-\frac{1}{2}\left(a^{2}+b^{2}\right)\left(\frac{2}{x+2 y}+\frac{1}{y-|x|}+\frac{1}{x+y-|x|}\right)+O\left(r^{-2}\right), \\
y_{9}= & y+\frac{1}{2}\left(a^{2}+b^{2}\right)\left(\frac{1}{x+2 y}-\frac{\operatorname{sgn} x}{y-|x|}+\frac{1-\operatorname{sgn} x}{x+y-|x|}\right) \\
& +\left(\frac{x}{\left(x^{2}+a^{2}\right)^{1 / 2}}-\frac{x}{\left(x^{2}+b^{2}\right)^{1 / 2}}\right)\left(\rho_{1}+\rho_{4}\right) \\
& -\frac{1}{2}\left(a^{2}+b^{2}\right)\left(\frac{x}{\left(x^{2}+a^{2}\right)^{1 / 2}}-\operatorname{sgn} x\right)\left(\frac{1}{x+y-|x|}+\frac{1}{y-|x|}\right)+O\left(r^{-2}\right) .
\end{aligned}
$$

(4.29) satisfies the conditions of Theorem 4. Choose $p, q$ as in the preliminary discussion and

$$
\begin{aligned}
\hat{p}= & O\left(r^{-2}\right), \\
\hat{q}= & \left(\frac{x}{\left(x^{2}+a^{2}\right)^{1 / 2}}-\frac{x}{\left(x^{2}+b^{2}\right)^{1 / 2}}\right)\left(\rho_{1}+\rho_{4}\right) \\
& -\frac{1}{2}\left(a^{2}+b^{2}\right)\left(\frac{x}{\left(x^{2}+a^{2}\right)^{1 / 2}}-\operatorname{sgn} x\right)\left(\frac{1}{x+y-|x|}+\frac{1}{y-|x|}\right)+O\left(r^{-2}\right),
\end{aligned}
$$

on $K_{02}$. The corresponding expressions on the other cones may be obtained by direct calculation or by applying the mapping (4.4) to (4.29). It is easily seen that on $\tilde{K}_{0}(4.29)$ is identical with (4.6). Note that while $q+\hat{q}$ is analytic on $K_{02}, q$ and $\hat{q}$ are each discontinuous on the ray $x=0, y<0$.

The conditions (a), (b), (c) and (f) of Theorem 4 are easily verified. For example, $x q-y p \equiv \frac{3}{2}\left(a^{2}+b^{2}\right)$. All that remains to prove is

(d) $\int_{t_{1}}^{t_{2}} \hat{q}(A \cos t, A \sin t) d t=o\left(A^{-1}\right), A \rightarrow \infty$, and (e) $x \hat{q}-y \hat{p}=o(1)$ near infinity.

Let $\xi=A \cos t$. One of the terms in $\int \hat{q}(\xi, \eta) d t$ includes

$$
\int \xi\left(\xi^{2}+a^{2}\right)^{-1 / 2} d t=\int A \cos t\left(A^{2} \cos ^{2} t+a^{2}\right)^{-1 / 2} d t=\arcsin \left(\sin t\left(1+a^{2} / A^{2}\right)^{-1 / 2}\right) \text {. }
$$

By the inequality

$$
0 \leq \arcsin x-\arcsin y \leq \arcsin \left(x^{2}-y^{2}\right)^{1 / 2}, \quad 0 \leq y \leq x,
$$

we have

$$
\begin{aligned}
& \left|\int_{t_{1}}^{t_{2}}\left(\xi\left(\xi^{2}+a^{2}\right)^{-1 / 2}-\xi\left(\xi^{2}+b^{2}\right)^{-1 / 2}\right) d t\right| \\
& \quad=\left|\arcsin \left(\sin t\left(1+a^{2} / A^{2}\right)^{-1 / 2}\right)-\arcsin \left(\sin t\left(1+b^{2} / A^{2}\right)^{-1 / 2}\right)\right|_{t_{1}^{2}}^{t_{2}} \\
& \quad \leq \arcsin \left(\sin t\left(\frac{\left|b^{2}-a^{2}\right|}{A^{2}+a^{2}+b^{2}}\right)^{1 / 2}\right)\left(t_{2}\right)+\arcsin \left(\sin t\left(\frac{\left|b^{2}-a^{2}\right|}{A^{2}+a^{2}+b^{2}}\right)^{1 / 2}\right)\left(t_{1}\right) \\
& \quad=O\left(A^{-1}\right)
\end{aligned}
$$

The other term of $\int \hat{q}(\xi, \eta) d t$ includes a similar factor, with $a, b$ replaced by $a, 0$. 
Since $\rho_{4}+\rho_{1}=O\left(r^{-1}\right)$ and $-1 /(-y+|x|)-1 /(x+y-|x|)=O\left(r^{-1}\right),(\mathrm{d})$ is proved. In order to prove $(e)$, it suffices to verify that

$$
x\left(x /\left(x^{2}+a^{2}\right)^{1 / 2}-x /\left(x^{2}+b^{2}\right)^{1 / 2}\right)
$$

is bounded for every $x$, which is immediate.

The mapping $S=T^{9}$ also has the intersection property $(\mathrm{g}) . T$ is area preserving in the whole plane and has a unique finite fixed point

$$
\left.\left.\left(\left(4 a^{2}+b^{2}\right) / 15\right)^{1 / 2},\left(4 b^{2}+a^{2}\right) / 15\right)^{1 / 2}\right) .
$$

Every closed curve which surrounds infinity and lays in a small neighbourhood of infinity, surrounds the finite fixed point too, and so by the area-preserving property near the finite fixed point, it intersects its image curve. Thus, finally, we may conclude by Theorem 4 that the mapping (4.4) has invariant closed curves around infinity. (See figure 2.)

The occurrence of the finite fixed point of (4.4) is not accidental. By [8], if $f:\left\{x \in R^{n} ;\|x\| \geq r\right\} \rightarrow R^{n}$ is continuous such that for every boundary point $y, \mid y \|=r$, there is no $m>1$ with $f(y)=m y$, then $f$ has a fixed point. Otherwise, $g(y)=$ $r(f(y)-y) /\|f(y)-y\|$ is continuous and by the Brouwer fixed point theorem there exists a point $y$ such that $g(y)=r(f(y)-y) /\|f(y)-y\|=y$. But then $\|y\|=r$ and $f(y)=y(1+\|f(y)-y\| / r)=m y$, where $m>1$, a contradiction. Now, if $x Q-y P \neq 0$ near infinity, then (3.1) satisfies the conditions of $[8]$ on a sufficiently large circle, so we have have:

If the mapping (3.1) is continuous and area preserving in the whole plane and $x Q-y P \neq 0$ near infinity, then it has the intersection property near infinity.

The existence of closed invariant curves of (4.4) near infinity also implies that every sequence of complex numbers which satifies (4.2), is bounded. Indeed, for given $z_{0}, z_{1}$, take $x=\operatorname{Re}\left\{z_{0}\right\}, y=\operatorname{Re}\left\{z_{1}\right\}, a=\operatorname{Im}\left\{z_{0}\right\}, b=\operatorname{Im}\left\{z_{1}\right\}$ and let (4.4) be the corresponding mapping. Choose an invariant curve $\Gamma$, sufficiently close to infinity, such that $(x, y)$ and the finite fixed point of $T$ are in its interior, int $(\Gamma)$. Since $\Gamma$ is invariant and $T$ is one to one and area preserving, int $(\Gamma)$ is invariant, too. So all the iterates $\left(x_{n}, y_{n}\right)$ lay in int $(\Gamma)$ and the sequence $z_{n}$ is bounded.

Acknowledgement. This research was supported by the Fund for the Promotion of Research at the Technion.

\section{REFERENCES}

[1] M. Brown. Problem 6439. Amer. Math. Monthly 90 (1983), 569; 92 (1985), 218.

[2] R. L. Devaney. A piecewise linear model for the zones of instability of an area-preserving mapping. Physica $10 D$ (1984), 387-393.

[3] C. Froeschle. Etude numérique de transformations ponctuelles planes conservant les aires. C.R. Acad. Sci. Paris 266 (1968), 747-749.

[4] M. Hénon. Numerical study of quadratic area-preserving mappings. Quarterly J. Applied Math. 27 (1969), 291-312.

[5] M. R. Herman. Sur les courbes invariantes par les difféomorphismes de l'anneau. $I$ : Astérisque 103-104 (1983), II: ibid. 144 (1986). 
[6] J. Moser. On invariant curves of area-preserving mappings of an annulus. Nachr. Akad. Wiss., Gottingen II, Math. Phys. K1. (1962), 1-20.

[7] J. Moser. Stability and nonlinear character of ordinary differential equations. In R. Langer ed., Nonlinear Problems, The University of Wisconsin Press, 1963.

[8] S. Reich. Problem 5721. Amer. Math. Monthly 77 (1970), 313; 78 (1971), 310.

[9] H. Russmann. Uber invariante Kurven differenzierbarer Abbildungen einen Kreisringes. Nachr. Akad. Wiss., Gottingen II, Math. Phys. K1. (1970), 67-105.

[10] C. L. Siegel \& J. Moser. Lectures on Celestial Mechanics. Springer Verlag, Berlin, 1971.

[11] C. Simo. Stability of degenerate fixed points of analytic area preserving mappings. Astérisque 98-99 (1982), 184-194. 